\title{
Нарушения ритма
}

\section{и проводимости сердца}

\section{при системной склеродермии}

\section{Алекперов Р.Т. • Черемухина Е.О. • Новикова Д.С. • Ананьева Л.П.}

Алекперов Ризван Таир оглы д-р мед. наук,

профессор кафедры терапии

факультета усовершенствования

врачей' ${ }^{1}$ ст. науч. сотр. ${ }^{2}$

$\triangle 129110$, г. Москва, ул. Щепкина, 61/2

Российская Федерация.

Тел.: +7 (495) 6816840.

E-mail: ralekperov@list.ru

\section{Черемухина Елена Олеговна -} аспирант ${ }^{2}$

Новикова Диана Сергеевна д-р мед. наук, ст. науч. сотр. 2

\section{Ананьева Лидия Петровна -}

д-р мед. наук, профессор, заведующая

лабораторией микроциркуляции

и воспаления ${ }^{2}$
Цель - определить частоту отдельных видов аритмий и нарушений проводимости сердца в российской популяции больных системной склеродермией (ССД) по данным стандартной и суточной электрокардиографии (ЭКГ) и оценить корреляции этих изменений с клиническими параметрами заболевания.

Материал и методы. В исследование были включены 80 больных ССД и 60 сопоставимых с ними по полу и возрасту условно здоровых лиц, составивших контрольную группу. Всем участникам исследования проводили стандартную ЭКГ в покое и суточное холтеровское мониторирование (ХМ) ЭКГ.

Результаты. При стандартной ЭКГ у больных ССД нарушения ритма определялись в $14(17,5 \%)$ случаях, в том числе синусовая аритмия - у 2 больных и экстрасистолия у 13 (16\%). Частота наджелудочковой и желудочковой экстрасистолии составила 5 (6\%) и 7 (9\%) случаев соответственно; в 1 наблюдении отмечалось сочетание наджелудочковых и желудочковых экстрасистол. У 9 (11\%) больных ССД выявлялись ЭКГ-признаки очагового фиброза миокарда различной локализации. ХМ ЭКГ показало высокую частоту наджелудочковых и желудочковых экстрасистол, которые выявлялись у 40 и 65\% больных ССД соответственно

Частота и выраженность аритмий сердца у больных ССД была достоверно больше, чем в контрольной группе. У $36 \%$ больных отмечаются потенциально опасные в отношении развития злокачественной аритмии и внезапной сердечной смерти желудочковые экстрасистолы высокой градации.

Заключение. Нарушения ритма и проводимости сердца развиваются у большинства больных ССД. Стандартная ЭКГ не отражает истинную частоту аритмий сердца у больных ССД. Суточное ХМ ЭКГ является обязательным методом обследования больных ССД и контроля эффективности лечения.

Ключевые слова: системная склеродермия, аритмия, блокады сердца, холтеровское мониторирование, электрокардиография.

'ГБУЗ МО «Московский областной научно-исследовательский клинический институт им. М.Ф. Владимирского»; 129110, г. Москва, ул. Щепкина, 61/2, Российская Федерация

${ }^{2}$ ФГБНУ «Научно-исследовательский институт ревматологии им. В.А. Насоновой»; 115522, г. Москва, Каширское шоссе, 34А, Российская Федерация 


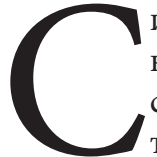

истемная склеродермия (ССД) - хроническое аутоиммунное заболевание соединительной ткани, которое характеризуется распространенными нарушениями микроциркуляции, фиброзной трансформацией кожи и внутренних органов [1]. Согласно современным представлениям, основу патогенеза заболевания составляют: 1) активация и повреждение сосудистых эндотелиальных клеток; 2) избыточная продукция компонентов внеклеточного матрикса активированными фибробластами; 3) дисбаланс клеточной и гуморальной иммунной системы [2]. Комбинация этих факторов клинически проявляется синдромом Рейно, ишемическими язвами кожи, нарушениями функций легких, сердца, желудочно-кишечного тракта и почек разной степени выраженности.

Несмотря на очевидные успехи последних двух десятилетий в области лечения ССД выживаемость больных ССД остается наиболее низкой среди всех системных заболеваний соединительной ткани. Так, по данным, полученным в ходе многих исследований, 5- и 10-летняя выживаемость больных ССД варьирует в пределах 73-90 и $60-80 \%$ соответственно $[3,4,5,6,7]$, а средняя продолжительность жизни после постановки диагноза составляет 12 лет [8]. В структуре причин смерти больных ССД основное место занимают поражения легких и сердца. При этом летальность вследствие поражения сердца сопоставима с частотой летальных исходов из-за поражения легких и составляет, по разным данным, от 20 до 36\% всех смертей, причина которых непосредственно связана с ССД [7, 9]. Поражение сердца является неблагоприятным прогностическим признаком и ассоциируется с низкой выживаемостью больных ССД [10, 11]. Метаанализ данных 1645 больных ССД показал: относительный риск летального исхода при поражении сердца составляет 2,8 (95\% доверительный интервал (ДИ) 2,1$3,8)$, что превышает аналогичный показатель при поражениях легких и почек - 1,6 (95\% ДИ 1,3-2,2) и 1,9 (95\% ДИ 1,4-2,5) соответственно [11]. Вместе с тем поражение сердца при ССД часто остается нераспознанным или неадекватно оцененным изза неспецифичности симптомов и малой доступности неинвазивных методов ранней диагностики изменений в сердце $[12,13]$.

При ССД могут поражаться все структуры сердца, клиническая и прогностическая значимость которых существенно различаются. Наибольшую опасность представляют склеродермическая кардиомиопатия с диастолической дисфункцией левого желудочка и нарушения ритма, так как эти клинические варианты ассоциируются с очень плохим жизненным прогнозом больных ССД [14]. Нарушения ритма могут быть жизнеугрожающими из-за высокого риска внезапной сердечной смерти [15], частота которой при ССД может достигать 13\% [16]. Исследования у больных с развернутой клинической картиной ССД выявили изменения ЭКГ почти в половине случаев $[17,18]$. Эти изменения включали нарушения проводимости, неспецифические изменения сегмента ST-T, гипертрофию правого или левого желудочков, инфарктоподобные изменения. В исследовании GENISOS (Genetics versus Environment in Scleroderma Outcome Study) было показано, что клинически значимые аритмии, выявляемые при ЭКГ, наряду с другими 6 параметрами (возраст более 65 лет, форсированная жизненная емкость легких менее $50 \%$ от должной величины, отсутствие антицентромерных антител, артериальная гипертония, фиброз легких при рентгенографии и сниженный индекс массы тела) являются независимым предиктором летальности больных ССД [19].

Целью настоящего исследования было определить частоту отдельных видов аритмий и нарушений проводимости сердца в российской популяции больных по данным стандартной и суточной ЭКГ и оценить корреляции этих изменений с клиническими параметрами ССД.

\section{Материал и методы}

В исследование были включены 80 больных ССД (73 женщины и 7 мужчин) в возрасте от 21 до 80 лет и длительностью болезни от 1 года до 30 лет. У всех больных диагноз соответствовал критериям Американской коллегии ревматологов (критерии ACR, 1980) [20]. У большинства больных (51 из 80) определялась лимитированная форма ССД, согласно классификации E.C. LeRoy и соавт. [21]. У всех пациентов были выполнены стандартная ЭКГ в покое и суточное холтеровское мониторирование (XM) ЭКГ, рентгенография или компьютерная томография органов грудной клетки. Кроме того, у большинства больных были исследованы функция внешнего дыхания и диффузионная способность легких, проведена допплеровская эхокардиография с расчетом систолического давления в легочной артерии. Анализ стандартной ЭКГ и ХМ ЭКГ проводился опытным кардиологом, который не был осведомлен о состоянии пациента и выраженности болезни.

Клиническая характеристика больных включала следующие параметры. 
1. Пол, n (\%)

- женский - 7 (9);

- мужской - 73 (91).

2. Возраст, годы, $-52,8 \pm 11,5(53)^{*}$.

3. Длительность ССД, годы, $-9,5 \pm 8,2(6,5)^{*}$.

4. Форма ССД, n (\%)

- диффузная - 29 (36);

- лимитированная - 51 (64).

5. Жалобы, n (\%)

- кардиалгия - 13 (16);

- перебои в сердце - 21 (26);

- одышка - 44 (55).

6. Класс одышки по NYHA ${ }^{* *}, \mathrm{n}(\%)$

- 1-й - 36 (45);

- 2-й - $32(40)$;

- 3-й - 12 (15);

$-4-\breve{и}-0$.

7. Форсированная жизненная емкость легких менее 80\%, n (\%), - 17 (27) из 64.

8. Диффузионная способность легких менее $80 \%, \mathrm{n}(\%),-52$ (88) из 59.

9. Систолическое давление в легочной артерии более 35 мм рт. ст., n (\%), - 19 (31) из 61.

10. Дисфункция левого желудочка, n (\%), 39 (49).

11. Лечение, $\mathrm{n}(\%)$

- глюкокортикостероиды - 50 (63);

- цитотоксические препараты или иммунодепрессанты - 22 (28);
- нестероидные противовоспалительные препараты - 9 (11);

- кальциевые блокаторы: нифедипин / верапамил - 32 (40) / 9 (11);

- ингибиторы ангиотензинпревращающего фермента - 8 (10);

- бета-адреноблокаторы - 22 (28);

- нитраты - 4 (5);

- статины - 31 (39).

Контрольную группу составили 60 человек в возрасте от 23 до 60 лет из числа медицинского и технического персонала ФГБНУ «Научно-исследовательский институт ревматологии им. В.А. Насоновой». Контрольная группа была сопоставима с группой больных ССД по полу (6 мужчин и 54 женщины) и возрасту $(49,4 \pm 6,8$ года, медиана - 50,0 года). Всем включенным в контрольную группу выполняли стандартную ЭКГ и ХМ ЭКГ.

Для статистической обработки результатов применялись непараметрические методы анализа. Сравнение количественных параметров между группами проводили с применением U-критерия Манна - Уитни. Корреляционные связи переменных изучали методом ранговой корреляции Спирмена. Для сравнения качественных параметров между группами применяли двусторонний точный критерий Фишера. Различия считались достоверными при значении $\mathrm{p}<0,05$.
* Данные представлены в виде среднего значения и стандартного отклонения $(\mathrm{M} \pm \mathrm{SD})$, в скобках указана медиана (Me).

** По классификации Нью-Йоркской кардиологической ассоциации (New York Heart Association - NYHA).

Таблица 1. Частота и выраженность различных типов аритмий у больных ССД в сравнении с контролем

\begin{tabular}{|c|c|c|c|}
\hline Параметр & Больные ССД & Контрольная группа & $\mathrm{p}$ \\
\hline Частота НЖЭ, n (\%) & $32(40)$ & $5(8)$ & $<0,001$ \\
\hline Количество НЖЭ* & $926 \pm 2705$ & $219 \pm 1032$ & $<0,001$ \\
\hline Частота одиночных НЖЭ, n (\%) & $30(37,5)$ & $5(8)$ & $<0,001$ \\
\hline Количество одиночных НЖЭ* & $729 \pm 2249$ & $214 \pm 1029$ & $<0,001$ \\
\hline Частота парных НЖЭ, n (\%) & $44(55)$ & $13(21)$ & $<0,001$ \\
\hline Количество парных НЖЭ* & $28 \pm 85$ & $0,88 \pm 3,1$ & $<0,001$ \\
\hline Частота пробежек НЖТ, n (\%) & $35(44)$ & $13(21)$ & $<0,01$ \\
\hline Количество пробежек НЖТ* & $28 \pm 154$ & $0,7 \pm 3,3$ & $<0,01$ \\
\hline Частота ЖЭ, n (\%) & $52(65)$ & $15(25)$ & $<0,001$ \\
\hline Количество ЖЭ* & $548 \pm 2232$ & $33 \pm 142$ & $<0,001$ \\
\hline Количество одиночных ЖЭ* & $372 \pm 1146$ & $33 \pm 142$ & $<0,001$ \\
\hline Частота парных ЖЭ, n (\%) & $12(15)$ & $1(1,6)$ & $=0,016$ \\
\hline Количество парных ЖЭ* & $15 \pm 94$ & $0,01 \pm 0,12$ & нд \\
\hline Частота пробежек ЖТ, n (\%) & $8(10)$ & $1(1,6)$ & нд \\
\hline Количество пробежек ЖТ* & $1,1 \pm 0,3$ & $0,01 \pm 0,12$ & $<0,001$ \\
\hline Частота сочетанных НЖЭ и ЖЭ, n (\%) & $50(63)$ & $7(12)$ & $<0,001$ \\
\hline
\end{tabular}

НЖЭ - наджелудочковые экстрасистолы, НЖТ - наджелудочковая тахикардия, ЖЭ - желудочковые экстрасистолы, ЖТ - желудочковая тахикардия, ССД - системная склеродермия, нд - различия статистически не достоверны

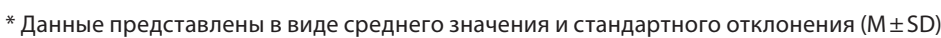


Таблица 2. Частота отдельных градаций ЖЭ по классификации Лауна, n (\%)

\begin{tabular}{lll}
\hline Класс ЖЭ & Больные ССД & Контрольная группа \\
\hline 0 (ЖЭ отсутствуют) & $28(35)$ & $45(75)$ \\
I (редкие монотопные ЖЭ менее 30 в час) & $17(21)$ & $10(17)$ \\
II (частые монотопные ЖЭ более 30 в час) & $6(8)$ & $1(2)$ \\
III (политопные ЖЭ) & $16(20)$ & $1(2)$ \\
IVa (монотопные парные ЖЭ) & $4(5)$ & $1(2)$ \\
IVb (политопные парные ЖЭ) & $1(1)$ & - \\
V (пробежки желудочковой тахикардии) & $8(10)$ &
\end{tabular}

ЖЭ - желудочковые экстрасистолы, ССД - системная склеродермия

Статистический анализ проводился с применением пакета программ Statistica 6.0.

\section{Результаты}

Стандартная ЭКГ, выполненная в покое, выявила нарушения проводимости у 27 (34\%) больных ССД. Наиболее часто регистрировали блокаду левой ножки пучка Гиса - у 15 (19\%) из 80 больных. Блокада правой ножки пучка Гиса отмечена у 7 (9\%) больных. В 5 (6\%) случаях наблюдалась бифасцикулярная блокада. Нарушения ритма по данным ЭКГ определялись всего в 14 (17,5\%) случаях, в том числе синусовая аритмия - у 2 больных и экстрасистолия - у 13 (16\%). Частота наджелудочковой и желудочковой экстрасистолии была приблизительно одинаковой: 5 (6\%) и 7 (9\%) наблюдений соответственно; в 1 случае зафиксировали сочетание наджелудочковых и желудочковых экстрасистол. У 9 (11\%) больных выявлены ЭКГ-признаки очагового фиброза миокарда различной локализации.

ХМ ЭКГ показало достоверно более высокую частоту нарушений ритма сердца по сравнению со стандартной ЭКГ ( $<<0,001$ для всех показателей) (см. рисунок). Частота и выраженность аритмий сердца у больных ССД были статистически значимо больше, чем в контрольной группе (табл. 1). При этом у $36 \%$ больных отмечаются потенциально опасные в отношении развития злокачественной аритмии и внезапной сердечной смерти желудочковые экстрасистолы (ЖЭ) высокой градации по классификации Лауна [22] (табл. 2).

Сравнение между разными подгруппами больных ССД показало, что у пациентов с диффузной формой заболевания достоверно больше, чем при лимитированной форме, общее количество ЖЭ $(1268 \pm 3696$ и $175 \pm 487$ соответственно, $\mathrm{p}=0,03)$, а также количество одиночных ЖЭ (786 1805 и $149 \pm 404$ соответственно, $\mathrm{p}=0,016)$. Не было выявлено различий частоты отдельных типов аритмий между больными с длительностью болезни до 10 лет и более 10 лет. У больных старше 50 лет по сравнению с пациентами более молодого возраста наблюдалось большее количество наджелудочковых экстрасистол (НЖЭ) как в целом (1344 23276 и $165 \pm 575$ соответственно), так и по отдельности - одиночных $(1048 \pm 2721$ и $132 \pm 503$ соответственно) и парных $(39 \pm 100$ и 8,0 34 соответственно). Характерно, что длительность ССД у больных старше 50 лет была достоверно больше, чем у больных моложе этого возраста $(10,4 \pm 9,0$ и 7,5 $\pm 7,32$ года соответственно, $\mathrm{p}=0,017)$.

Следует иметь в виду, что значительная часть больных принимали антиаритмические препараты, а также лекарственные средства, оказывающие аритмогенное и кардиотоксическое действие, что, несомненно, влияло на истинную частоту нарушений ритма и проводимости сердца у включенных в исследование пациентов. Однако этот вопрос требует отдельного изучения с использованием соответствующих методов статистического анализа.

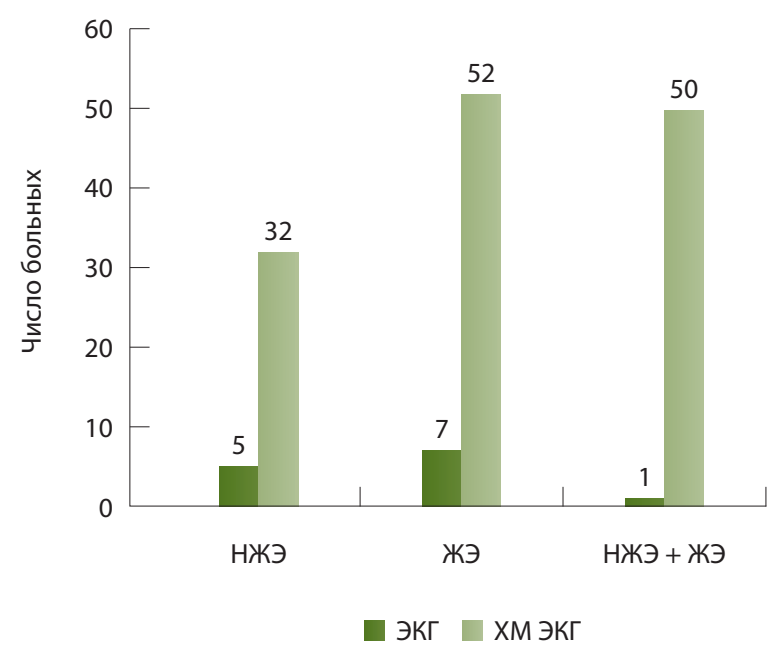

Частота нарушений ритма сердца у больных ССД по данным стандартной ЭКГ и ХМ ЭКГ; НЖЭ - наджелудочковые экстрасистолы, ЖЭ - желудочковые экстрасистолы 


\section{Обсуждение}

ССД представляет собой системное заболевание с множественными висцеральными поражениями. Хотя высокая частота поражений сердца при ССД была установлена относительно недавно, в настоящее время уже общепризнано, что этот фактор является предвестником неблагоприятного исхода $[23,24]$. В нашей работе показана высокая частота аритмий, в том числе потенциально жизнеугрожающих. Аналогичные результаты были получены и другими исследователями. Так, С. Ferri и соавт. по данным стандартной ЭКГ выявили нарушения проводимости, неспецифические изменения сегмента ST-T или нарушения ритма у $30 \%$ больных, тогда как при суточном ХМ ЭКГ наджелудочковые аритмии определялись у 66\% больных, а желудочковые аритмии - у 90\% (в том числе полиморфные ЖЭ у $40 \%$, парные экстрасистолы - у $28 \%$ и 1 или более пробежек желудочковой тахикардии - у 13\%) [25]. Учитывая ассоциацию аритмий с неблагоприятным исходом и низкую чувствительность стандартной ЭКГ, представляется необходимым проведение кардиологического обследования с целью ранней диагностики изменений ЭКГ даже больным ССД без симптомов поражения сердца. Обоснованность такого подхода признается и другими авторами [26].

У значительной доли больных ССД отмечаются различные нарушения проводимости, хотя и с меньшей частотой, чем нарушения ритма. В нашем исследовании блокады левой и/или правой ножек пучка Гиса наблюдались у трети больных с некоторым (не достигшим уровня статистической значимости) преобладанием блокады левой ножки пучка Гиса. Интересно, что сходные результаты были получены в работе, выполненной в 1981 г. [27]. В этом проспективном исследовании по данным ЭКГ в покое нарушения проводимости выявлялись у $32 \%$ больных ССД (16 из 50). Наиболее частыми изменениями были блокада левой ножки пучка Гиса (16\%) и атриовентрикулярная блокада 1-й степени (8\%), тогда как блокады 2-й и 3-й степени встречались редко $(<2 \%)$.

Таким образом, аритмии и нарушения проводимости являются частым и важным проявлением поражений сердца при ССД. Эти нарушения могут быть выражены в разной степени и способны стать причиной летального исхода. Хотя механизмы, лежащие в основе этих нарушений, не вполне ясны, именно поражение миокарда и фиброз рассматривают в качестве наиболее значимых аритмогенных факторов. Раннее выявление угрожающих жизни желудочковых аритмий может стать ключевым условием для улучшения жизненного прогноза больных ССД.

\section{Выводы}

1. Нарушения ритма и проводимости сердца развиваются у большинства больных ССД.

2. Стандартная ЭКГ не отражает истинной частоты аритмий сердца у больных ССД.

3. Суточное ХМ ЭКГ является обязательным методом обследования больных ССД и контроля эффективности лечения. (;)

\section{Литература (References)}

1. Гусева НГ. Системная склеродермия и псевдосклеродермические синдромы. М.: Медицина; 1993. 300 с.

(Guseva NG. Systemic sclerosis and pseudoscleroderma syndromes. Moscow: Meditsina; 1993. 300 p. Russian).

2.Geyer M, Müller-Ladner U. The pathogenesis of systemic sclerosis revisited. Clin Rev Allergy Immunol. 2011;40(2):92-103.

3. Abu-Shakra M, Lee P. Mortality in systemic sclerosis: a comparison with the general population. J Rheumatol. 1995;22(11):2100-2.

4. Jacobsen S, Halberg P, Ullman S. Mortality and causes of death of 344 Danish patients with systemic sclerosis (scleroderma). Br J Rheumatol. 1998;37(7):750-5.

5. Bryan C, Knight C, Black CM, Silman AJ. Prediction of five-year survival following presenta- tion with scleroderma: development of a simple model using three disease factors at first visit. Arthritis Rheum. 1999;42(12):2660-5.

6. Simeón CP, Armadans L, Fonollosa V, Solans R, Selva A, Villar M, Lima J, Vaqué J, Vilardell M. Mortality and prognostic factors in Spanish patients with systemic sclerosis. Rheumatology (Oxford). 2003;42(1):71-5.

7. Al-Dhaher FF, Pope JE, Ouimet JM. Determinants of morbidity and mortality of systemic sclerosis in Canada. Semin Arthritis Rheum. 2010;39(4):269-77.

8. Mayes MD. Scleroderma epidemiology. Rheum Dis Clin North Am. 2003;29(2):239-54.

9. Ferri C, Valentini G, Cozzi F, Sebastiani M, Michelassi C, La Montagna G, Bullo A, Cazzato M, Tirri E, Storino F, Giuggioli D, Cuomo G, Rosada M, Bombardieri S, Todesco S, Tirri G; Sys- temic Sclerosis Study Group of the Italian Society of Rheumatology (SIR-GSSSc). Systemic sclerosis: demographic, clinical, and serologic features and survival in 1,012 Italian patients. Medicine (Baltimore). 2002;81(2):139-53.

10. Medsger TA Jr, Masi AT, Rodnan GP, Benedek TG, Robinson $H$. Survival with systemic sclerosis (scleroderma). A life-table analysis of clinical and demographic factors in 309 patients. Ann Intern Med. 1971;75(3):369-76.

11. loannidis JP, Vlachoyiannopoulos PG, Haidich $A B$, Medsger TA Jr, Lucas M, Michet CJ, Kuwana M, Yasuoka $H$, van den Hoogen $F$, Te Boome L, van Laar JM, Verbeet NL, Matucci-Cerinic M, Georgountzos A, Moutsopoulos HM. Mortality in systemic sclerosis: an international meta-analysis of individual patient data. Am J Med. 2005;118(1):2-10. 
12. Meune C, Vignaux $\mathrm{O}$, Kahan A, Allanore $\mathrm{Y}$. Heart involvement in systemic sclerosis: evolving concept and diagnostic methodologies. Arch Cardiovasc Dis. 2010;103(1):46-52.

13. Desai CS, Lee DC, Shah SJ. Systemic sclerosis and the heart: current diagnosis and management. Curr Opin Rheumatol. 2011;23(6): 545-54.

14. Marasini B, Massarotti M, Cossutta R. Scleroderma heart disease. Int J Immunopathol Pharmacol. 2005;18(4):609-14.

15. Seferović PM, Ristić AD, Maksimović R, Simeunović DS, Ristić GG, Radovanović G, Seferović D, Maisch B, Matucci-Cerinic M. Cardiac arrhythmias and conduction disturbances in autoimmune rheumatic diseases. Rheumatology (Oxford). 2006;45 Suppl 4:iv39-42.

16. Bulkley BH, Ridolfi RL, Salyer WR, Hutchins GM. Myocardial lesions of progressive systemic sclerosis. A cause of cardiac dysfunction. Circulation. 1976;53(3):483-90.

17. Follansbee WP, Curtiss El, Rahko PS, Medsger TA Jr, Lavine SJ, Owens GR, Steen VD. The electrocardiogram in systemic sclerosis (scleroderma). Study of 102 consecutive cases with functional correlations and review of the literature. Am J Med. 1985;79(2):183-92.
18. Morelli S, Sgreccia A, Ferrante L, Barbieri C, Bernardo ML, Perrone C, De Marzio P. Relationships between electrocardiographic and echocardiographic findings in systemic sclerosis (scleroderma). Int J Cardiol. 1996;57(2):151-60.

19. Assassi S, Del Junco D, Sutter K, McNearney TA, Reveille JD, Karnavas A, Gourh P, Estrada-Y-Martin RM, Fischbach M, Arnett FC, Mayes MD. Clinical and genetic factors predictive of mortality in early systemic sclerosis. Arthritis Rheum. 2009;61(10):1403-11.

20. Preliminary criteria for the classification of systemic sclerosis (scleroderma). Subcommittee for scleroderma criteria of the American Rheumatism Association Diagnostic and Therapeutic Criteria Committee. Arthritis Rheum. 1980;23(5):581-90.

21. LeRoy EC, Black C, Fleischmajer R, Jablonska S, Krieg T, Medsger TA Jr, Rowell N, Wollheim F. Scleroderma (systemic sclerosis): classification, subsets and pathogenesis. J Rheumatol. 1988;15(2):202-5.

22. Lown B, Wolf M. Approaches to sudden death from coronary heart disease. Circulation. 1971;44(1):130-42.

23. Komócsi A, Vorobcsuk A, Faludi R, Pintér T, Lenkey Z, Költo G, Czirják L. The impact of cardio- pulmonary manifestations on the mortality of SSc: a systematic review and meta-analysis of observational studies. Rheumatology (Oxford). 2012;51(6):1027-36.

24. Elhai M, Meune C, Avouac J, Kahan A, Allanore $\mathrm{Y}$. Trends in mortality in patients with systemic sclerosis over 40 years: a systematic review and meta-analysis of cohort studies. Rheumatology (Oxford). 2012;51(6):1017-26.

25. Ferri $C$, Bernini $L$, Bongiorni $M G$, Levorato $D$, Viegi G, Bravi P, Contini C, Pasero G, Bombardieri S. Noninvasive evaluation of cardiac dysrhythmias, and their relationship with multisystemic symptoms, in progressive systemic sclerosis patients. Arthritis Rheum. 1985;28(11):1259-66.

26. Rokas S, Mavrikakis M, Agrios N, Mylonas D, Antoniadou L, Moulopoulos S. Electrophysiologic abnormalities of cardiac function in progressive systemic sclerosis. J Electrocardiol. 1996;29(1):17-25.

27. Roberts NK, Cabeen WR Jr, Moss J, Clements PJ, Furst DE. The prevalence of conduction defects and cardiac arrhythmias in progressive systemic sclerosis. Ann Intern Med. 1981;94(1):38-40.

\title{
Cardiac arrhythmia and conduction disturbances in patients with systemic sclerosis
}

\author{
Alekperov R.T. • Cheremukhina E.O. • Novikova D.S. • Anan'eva L.P.
}

Aim: To assess prevalence of certain cardiac arrhythmias and conduction disturbances in the Russian population of systemic sclerosis patients using standard 12-lead and 24-hour electrocardiography (ECG); to estimate correlation between cardiac arrhythmia and clinical features of systemic sclerosis.

Materials and methods: 80 systemic sclerosis patients and 60 sex- and age-matched controls were included. All patients underwent standard 12-lead and 24-hour ECG.

Results: Arrhythmias on standard 12-lead ECG were demonstrated in 14 patients (17.5\%) including sinus arrhythmia in 2 cases and premature beats in 13 cases (16\%). Supraventricular (SV) and ventricular (V) ectopic beats were recorded in $5(6 \%)$ and 7 (9\%) patients, respectively; in one patient both SV and V ectopic beats were found. ECG signs of focal fibrosis were demonstrated in 9 patients (11\%). In 24-hour ECG, frequencies of SV and $\mathrm{V}$ ectopic beats were 40 and $65 \%$, respectively.

Compared to the controls, systemic sclerosis patients had significantly higher prevalence and severity of cardiac arrhythmia. $36 \%$ of patients had high grade ventricular premature beats, associated with potential risk of life-threatening arrhythmia and sudden cardiac death.

Conclusion: Cardiac arrhythmias and conduction disturbances are found in the majority of patients with systemic sclerosis. Standard ECG does not reflect true prevalence of cardiac arrhythmia in systemic sclerosis. In systemic sclerosis patients, 24-hour ECG is recommended as obligate method for initial examination and treatment efficacy control.

Key words: systemic sclerosis, arrhythmia, cardiac blocks, 24-hour ECG, electrocardiography.
Alekperov Rizvan Tair ogly - MD, PhD, Professor, Internal Diseases Department, Postgraduate Medical School', Senior Research Associate ${ }^{2}$

$\triangle 61 / 2$ Shchepkina ul., Moscow, 129110 , Russian Federation. Tel.: +7 (495) 6816840. E-mail: ralekperov@list.ru

Cheremukhina Elena Olegovna - Fellow ${ }^{2}$

Novikova Diana Sergeevna - PhD, Senior Research Associate ${ }^{2}$

Anan'eva Lidiya Petrovna - PhD, Professor, Head of Laboratory of Microcirculation and Inflammation ${ }^{2}$

${ }^{1}$ Moscow Regional Research and Clinical Institute (MONIKI); 61/2 Shchepkina ul., Moscow, 129110, Russian Federation

${ }^{2}$ V.A. Nasonova Research Institute of Rheumatology, Russian Academy of Medical Sciences; 34A Kashirskoe shosse, Moscow, 115522, Russian Federation 